\title{
Silibinin and indocyanine green-loaded nanoparticles inhibit the growth and metastasis of mammalian breast cancer cells in vitro
}

\author{
Hui-ping SUN ${ }^{1,2}$, Jing-han $\mathrm{SU}^{1}$, Qing-shuo MENG ${ }^{1}$, Qi YIN ${ }^{1}$, Zhi-wen ZHANG ${ }^{1}$, Hai-jun YU ${ }^{1}$, Peng-cheng ZHANG ${ }^{1}$, \\ Si-ling $\mathrm{WANG}^{1, *}$, Ya-ping $\mathrm{LI}^{2, *}$ \\ ${ }^{1}$ School of Pharmacy, Shenyang Pharmaceutical University, Shenyang 110016, China; ${ }^{2}$ State Key Laboratory of Drug Research \& \\ Center of Pharmaceutics, Shanghai Institute of Materia Medica, Chinese Academy of Sciences, Shanghai 201203, China
}

\begin{abstract}
Aim: To improve the therapeutic efficacy of cancer treatments, combinational therapies based on nanosized drug delivery system (NDDS) has been developed recently. In this study we designed a new NDDS loaded with an anti-metastatic drug silibinin and a photothermal agent indocyanine green (ICG), and investigated its effects on the growth and metastasis of breast cancer cells in vitro. Methods: Silibinin and ICG were self-assembled into PCL lipid nanoparticles (SIPNs). Their physical characteristics including the particle size, zeta potential, morphology and in vitro drug release were examined. 4T1 mammalian breast cancer cells were used to evaluate their cellular internalization, cytotoxicity, and their influences on wound healing, in vitro cell migration and invasion.

Results: SIPNs showed a well-defined spherical shape with averaged size of $126.3 \pm 0.4 \mathrm{~nm}$ and zeta potential of $-10.3 \pm 0.2 \mathrm{mV}$. NIR laser irradiation substantially increased the in vitro release of silibinin from the SIPNs $(58.3 \%$ at the first $8 \mathrm{~h}$, and $97.8 \%$ for the total release). Furthermore, NIR laser irradiation markedly increased the uptake of SIPNs into 4T1 cells. Under the NIR laser irradiation, both SIPNs and IPNs (PCL lipid nanoparticles loaded with ICG alone) caused dose-dependent ablation of 4T1 cells. The wound healing, migration and invasion experiments showed that SIPNs exposed to NIR laser irradiation exhibited dramatic in vitro anti-metastasis effects.
\end{abstract}

Conclusion: SIPNs show temperature-sensitive drug release following NIR laser irradiation, which can inhibit the growth and metastasis of breast cancer cells in vitro.

Keywords: breast cancer; 4T1 mammalian breast cancer cells; metastasis; nanoparticles; silibinin; photothermal therapy; NIR laser irradiation

Acta Pharmacologica Sinica (2016) 37: 941-949; doi: 10.1038/aps.2016.20; published online 2 May 2016

\section{Introduction}

Breast cancer is the leading cause of cancer-related deaths among women in the world ${ }^{[1]}$, and the metastasis of the cancerous cells is responsible for over $90 \%$ of the deaths of these cancer patients ${ }^{[2]}$. Currently, chemotherapy remains the primary approach used to inhibit the growth and metastasis of tumors ${ }^{[3]}$. Although chemotherapy can successfully inhibit the growth of a primary tumor, it is often ineffective at preventing the spread or metastasis of cancer cells and the tumor recurrence $^{[4,5]}$. To inhibit the metastasis of cancer cells directly and efficiently, anti-metastasis drugs such as silibinin ${ }^{[6]}$ or

\footnotetext{
${ }^{*}$ To whom correspondence should be addressed.

E-mail silingwang@syphu.edu.cn (Si-ling WANG); ypli@simm.ac.cn (Ya-ping LI)

Received 2016-01-25 Accepted 2016-03-08
}

atorvastatin calcium ${ }^{[7]}$ have been investigated. Unfortunately, their clinical effects were not satisfactory due to the low drug delivery efficiency and bioavailability ${ }^{[8]}$. Therefore, the development of new strategies that prevent tumor cell growth and metastasis more efficiently remains a subject of great importance.

The nanosized drug delivery system (NDDS) is a promising strategy for increasing the accumulation of drugs in the tumor because of its enhanced penetration and retention (EPR) effects and for minimizing side effects ${ }^{[9-11]}$. To further improve the therapeutic efficacy of the cancer treatments, a combinational therapy based on the NDDS has been developed, which includes the chemo/photodynamic ${ }^{[12]}$ or the immuno/photothermal combinations ${ }^{[13]}$. The use of near-infrared (NIR) laser (650-900 nm) mediated photothermal therapy (PTT) in cancer therapy has recently gained more attention ${ }^{[14]}$. The NIR laser 
could induce hyperthermia that could cause the ablation of cancer cells and tumor organs with excellent transmissivity in deep tissues and minimal skin absorbance ${ }^{[15,16]}$. It exhibits higher targeting efficiency of the tumor than chemotherapy with greater spatial-temporal selectivity ${ }^{[17]}$. Moreover, NIRinduced hyperthermia has been applied to spatiotemporally control the release of drugs with site-selectivity, improve therapeutic efficacy and decrease side effects ${ }^{[18]}$. All of these have utilized the combination of PTT and chemotherapy for cancer therapy ${ }^{[19]}$. Numerous photothermal agents such as indocyanine green $(\mathrm{ICG})^{[20]}$, gold nanomaterials ${ }^{[21,22]}$ or carbon nanotubes ${ }^{[23]}$ have been explored. However, the outcome has still been focused on the treatment of primary tumors, and the direct inhibition of the metastasis has been ignored ${ }^{[14,16]}$. There have been no reports to date that investigate the potentially synergistic effects of NDDS, which combines PTT with antimetastasis drugs, on reducing tumor growth and metastasis together. Therefore, we designed a new NDDS, combining the anti-metastasis drug (silibinin) and the PTT agent to prevent tumor cell growth and metastasis simultaneously in vitro. Briefly, the silibinin and the ICG were self-assembled into the poly(caprolactone) (PCL) lipid nanoparticles (PNs) prepared by PCL, soybean phosphatidylcholine (SPC) and pluronic copolymer F68 (SIPNs). The 4T1 breast cancer cell line, which has a high metastatic capability ${ }^{[2]}$, was used for this study. The drug release was evaluated in vitro. The cellular internalization, cytotoxicity, wound healing assay, migration and invasion assays were carried out in $4 \mathrm{~T} 1$ cells. It was expected that the SIPNs would be effective at inducing the combined therapeutic effects of the anti-metastasis drug and the photothermal agents for the effective inhibition of cancer cell growth and metastasis in vitro.

\section{Materials and methods Materials}

The silibinin was purchased from the Aladdin Reagent Co Ltd (purity $\geq 98 \%$, Shanghai, China). The indocyanine green (ICG), sulforhodamine B (SRB) and Hoechst 33342 were purchased from Sigma-Aldrich (St Louis, MO, USA). The poly(caprolactone) (PCL) with ester endcap $\left(M_{n} 5000, M_{\mathrm{w}}\right.$ 10000) was purchased from the PolySci Tech ${ }^{\circledR}$ Co Ltd (Indiana, USA). The pluronic F68 was purchased from BASF Ltd (Shanghai, China). The soybean phosphatidylcholine (S100, SPC) was obtained from Lipoid GmbH (Ludwigshafen, Germany). The fetal bovine serum (FBS) and phosphate buffer solution (PBS) were obtained from Gibco-BRL (Burlington, Canada). The Nile red, the RPMI-1640 medium and the antibiotics were purchased from Invitrogen (Oregon, USA). All the chemical reagents were of analytical grade and were used without further purification.

\section{Cell culture}

The 4T1 mammary breast cancer cell line was obtained from the cell bank of Shanghai, Chinese Academy of Sciences (CAS, Shanghai, China) and were grown in RPMI-1640 (Gibco, USA) with $10 \%$ fetal bovine serum (FBS), $100 \mathrm{U} / \mathrm{mL}$ penicillin $\mathrm{G}$ sodium, $100 \mu \mathrm{g} / \mathrm{mL}$ streptomycin sulfate, $2.5 \mathrm{~g} / \mathrm{L}$ glucose and $0.11 \mathrm{~g} / \mathrm{L}$ sodium pyruvate. The cells were incubated at $37^{\circ} \mathrm{C}$ in a humidified incubator with $5 \% \mathrm{CO}_{2}$.

\section{The preparation and characterization of SIPNs}

In order to prepare the silibinin/ICG-loaded PCL/lipid nanoparticles (SIPNs), PCL (5.0 mg), SPC (5.0 mg) and silibinin $(0.5 \mathrm{mg})$ were first dissolved in acetone $(2 \mathrm{~mL})$ at $37^{\circ} \mathrm{C}$ to ensure that all the materials and drugs were in the organic phase. The ICG (0.2 mg) was dissolved in water and then added into the organic phase. Then, the final mixed solution was added into the aqueous solution containing F68 (0.5\%, $w / v)$ drop-wise under vigorous stirring. The mixed solution was further stirred using a high shear homogenizer (Panasonic, Japan) followed by stirring in the open air overnight at room temperature. The resulting solution was centrifuged at $5000 \times g$ for $20 \mathrm{~min}$. Then, the supernatant was saved and further centrifuged at $200000 \times g$ for $20 \mathrm{~min}$ to obtain the SIPNs.

The particle size and zeta potential of the SIPNs were determined by a Malven zetasizer ZEN3690 analyzer (Malven, UK; $n=3$ ). The morphology of the SIPNs was characterized by a transmission electron microscope (TEM) (JEOL JEM-1230, Tokyo, Japan). The drug loading efficiency and the encapsulation efficiency were both determined by HPLC assay $\left(C_{18}\right.$ column, $5 \mu \mathrm{m}, 4.6 \times 250 \mathrm{~mm}$, Waters, USA) with the mobile phase consisting of $0.1 \mathrm{~mol} / \mathrm{L}$ phosphate buffer $(\mathrm{pH} 3.0)$ and methanol $(1: 1, v / v)$. The column was kept at $40{ }^{\circ} \mathrm{C}$ with the detection wavelength set at $288 \mathrm{~nm}$. The thermal behaviors of the free ICG, SPNs, IPNs and SIPNs in PBS were detected under $808 \mathrm{~nm}$ laser irradiation (MDL-N-10W, Changchun New Industries, China) at $1.5 \mathrm{~W} \cdot \mathrm{cm}^{-2}$ for $8 \mathrm{~min}$, and the temperatures at different points were recorded by the accompanying software. The PBS was used as the negative control. The drug release profiles of the different nanoparticles including the SPNs, SIPNs and SIPNs with NIR laser irradiation at 1.5 $\mathrm{W} \cdot \mathrm{cm}^{-2}$ for $5 \mathrm{~min}$ were measured in PBS ( $\mathrm{pH}=7.4$ ) with $1 \%$ Tween- 80 by a dialysis method at $37^{\circ} \mathrm{C}$. An end-sealed dialysis bag $(\mathrm{MWCO}=3.5 \mathrm{kDa})$ was applied to load the $0.5 \mathrm{~mL}$ of the suspensions from the nanoparticles and was placed into $5 \mathrm{~mL}$ of PBS at $37^{\circ} \mathrm{C}$ while shaking at $100 \mathrm{r} / \mathrm{min}$ for $72 \mathrm{~h}$. At predetermined time points, including $0.5,1,2,4,8,24,48$ and $72 \mathrm{~h}$, the medium was withdrawn and replaced with an equal volume of fresh medium. Then, the silibinin was extracted with methanol and analyzed by HPLC.

\section{Cellular internalization experiment}

Nile red, which has a detectable autofluorescence, was used to replace the silibinin to visualize the internalization of the free silibinin, SPNs, SIPNs and SIPNs exposed to NIR laser irradiation. The $4 \mathrm{~T} 1$ cells were seeded onto 24 -well plates at a density of $2 \times 10^{4}$ cells and were incubated for $24 \mathrm{~h}$. The Nile red-loaded nanoparticles were prepared as described for the SIPNs above. The cells were then treated with the free Nile red, Nile red-loaded SPNs, SIPNs and SIPNs with NIR irradiation at $1.5 \mathrm{~W} \cdot \mathrm{cm}^{-2}$ for $5 \mathrm{~min}$ at the equivalent Nile red concentration of $2.0 \mu \mathrm{g} / \mathrm{mL}$ for $2 \mathrm{~h}$ at $37^{\circ} \mathrm{C}$, respectively. Afterwards, 
the cells were stained with Hoechst 33342 for an additional 30 min. After washing the treated cells with PBS three times, the cells were imaged using a fluorescence microscope (Olympus, Japan).

\section{In vitro cytotoxicity and photothermal ablation assays}

The 4T1 cells were seeded on 96-well plates at a density of $5 \times 10^{3}$ cells per well and were incubated for $24 \mathrm{~h}$. The medium was then replaced with fresh medium containing free silibinin, PNs, SPNs, IPNs with NIR irradiation at $1.5 \mathrm{~W} \cdot \mathrm{cm}^{-2}$ for $5 \mathrm{~min}$, SIPNs and SIPNs with NIR irradiation at $1.5 \mathrm{~W} \cdot \mathrm{cm}^{-2}$ for $5 \mathrm{~min}$ at different silibinin concentrations ranging from 1.0 to 100 $\mu \mathrm{g} / \mathrm{mL}$, ICG concentrations ranging from 0.3 to $30 \mu \mathrm{g} / \mathrm{mL}$ or PNs concentrations ranging from 0.023 to $2.3 \mathrm{mg} / \mathrm{mL}$ for $24 \mathrm{~h}$. The cells that did not receive any treatments were used as the controls. Cell viability was measured by the SRB assay. In brief, the cells were first fixed with $100 \mu \mathrm{L}$ of $10 \%$ trichloroacetic acid per well and incubated at $4^{\circ} \mathrm{C}$ for $1 \mathrm{~h}$. Then, the cells were stained with $35 \mu \mathrm{L}$ of $0.4 \%$ SRB for 20 min in the dark, and the free dye was removed with $1 \%(v / v)$ acetic acid three times. The protein-bound proteins were dissolved in $100 \mu \mathrm{L}$ of $10 \mathrm{mmol} / \mathrm{L}$ Tris base solution per well and the absorbance was measured at $560 \mathrm{~nm}$. Each assay was repeated six times.

For the photothermal ablation assay, the $4 \mathrm{~T} 1$ cells were seeded on 96-well plates at a density of $5 \times 10^{3}$ cells per well and incubated for $24 \mathrm{~h}$. The cells were further treated with fresh medium containing the different nanoparticles at a silibinin concentration of $50 \mu \mathrm{g} / \mathrm{mL}$ or an ICG concentration of 15 $\mu \mathrm{g} / \mathrm{mL}$ for $12 \mathrm{~h}$ in the dark. Then, the cells treated with IPNs and SIPNs with NIR irradiation were irradiated at $1.5 \mathrm{~W} \cdot \mathrm{cm}^{-2}$ for $5 \mathrm{~min}$ and incubated for another $12 \mathrm{~h}$. The cells treated with SIPNs were incubated in the dark for another $12 \mathrm{~h}$. Then, the live and dead cells were stained with calcein-AM (AM) and ethidium homodimer-1 (EthD-1) using the LIVE/ DEADViability/Cytotoxicity Kit (Life Technologies) and were detected using a fluorescence microscope (IX81, Olympus, Japan).

\section{Wound healing assay}

The 4T1 cells were seeded on 12-well plates at a density of $1 \times 10^{5}$ cells per well overnight until they reached $90 \%$ confluence. Then, the medium was removed, and a $200 \mu \mathrm{L}$ plastic pipette tip with a ruler was applied to scratch a vertical wound. Afterwards, the cells were washed with PBS three times, and fresh medium containing free silibinin, PNs, SPNs, IPNs with NIR, SIPNs and SIPNs exposed to NIR irradiation at a silibinin concentration of $10 \mu \mathrm{g} / \mathrm{mL}$ or an ICG concentration of $3.0 \mu \mathrm{g} / \mathrm{mL}$ for another $24 \mathrm{~h}$ was applied. A light microscope (Olympus, Japan) was used to image the cells before and after the $24 \mathrm{~h}$ incubation to detect the degree of wound healing. The cells that did not receive any treatments were used as the control.

\section{In vitro migration and invasion assay}

For the cell migration experiments, the $4 \mathrm{~T} 1$ cells were pretreated with the free silibinin, PNs, SPNs, IPNs with NIR,
SIPNs and SIPNs with NIR at the silibinin concentration of 10 $\mu \mathrm{g} / \mathrm{mL}$ or the ICG concentration of $3.0 \mu \mathrm{g} / \mathrm{mL}$ for $24 \mathrm{~h}$. Then, the cells were collected in the medium without serum, counted and seeded on the top chambers at a density of $1 \times 10^{5}$ cells in $100 \mu \mathrm{L}$ of serum-free medium. The lower chambers were filled with $500 \mu \mathrm{L}$ of complete medium containing 10\% FBS that was used as a chemoattractant. After they were incubated for another $24 \mathrm{~h}$, the upper medium was removed, and a cotton swab was applied to wash the cells that remained on the top chambers. Then, the transwell chambers were removed, fixed using $70 \%$ ethanol for $20 \mathrm{~min}$ and stained with cresyl violet for 20 min. Finally, the stained transwell chambers were visualized under a microscope, and images were taken. In order to further quantify the migration rates, the microscope was used to count the cells five times in random fields from each chamber. For the cell invasion experiment, matrigel was added to the upper chambers and incubated at $37^{\circ} \mathrm{C}$ for $12 \mathrm{~h}$. The cells seeded on the top chambers were at the density of $2 \times 10^{5}$. The protocol was then the same as described in the migration assay. The cells that did not receive any treatment were used as control. The migration and invasion rates were calculated using the control group.

\section{Statistical analysis}

The mean $\pm S D$ were determined for all the treatment groups. The statistical analysis was performed using Student's $t$-test (two-tailed). In $\left({ }^{*}\right)$ and $\left({ }^{* *}\right)$, the statistical significance was set at $P<0.05$ or $P<0.01$, respectively, when compared to the SIPNs exposed to NIR laser irradiation. In (\#) and (\#\#) the statistical significance was set at $P<0.05$ or $P<0.01$, respectively, when compared to free silibinin.

\section{Results}

The preparation and characterization of SIPNs

The silibinin and ICG were both loaded into the PCL/ lipid nanoparticles using the single-step nanoprecipitation method $^{[20]}$. Unencapsulated silibinin and ICG were removed by centrifugation. The average hydrodynamic diameter and zeta potential of SIPNs was $126.3 \pm 0.4 \mathrm{~nm}$ and $-10.3 \pm 0.2 \mathrm{mV}$, respectively (Figure 1A). The polydispersity index (PDI) of the nanoparticles was 0.203 . The negative charge of the nanoparticles could contribute to the suspension stability of the nanoparticles. The TEM images revealed that the SIPNs were successfully obtained (1) with a well-defined spherical shape displaying a narrow size distribution (Figure 1B), and (2) with a particle size that was consistent with the results detected by DLS, further indicating that the SIPNs were prepared as expected. The silibinin loading ratio and the encapsulation efficiency of the SIPNs were $4.2 \% \pm 0.3 \%$ and $97.5 \% \pm 0.4 \%$, respectively. The ICG loading ratio and the encapsulation efficiency of the SIPNs were $1.2 \% \pm 0.2 \%$ and $98.7 \% \pm 0.6 \%$, respectively.

To further determine whether the nanoparticles exhibited photothermal capabilities, photothermal conversion experiments were conducted to characterize the photothermal efficiency of the free ICG, SPNs, IPNs and SIPNs under laser irra- 

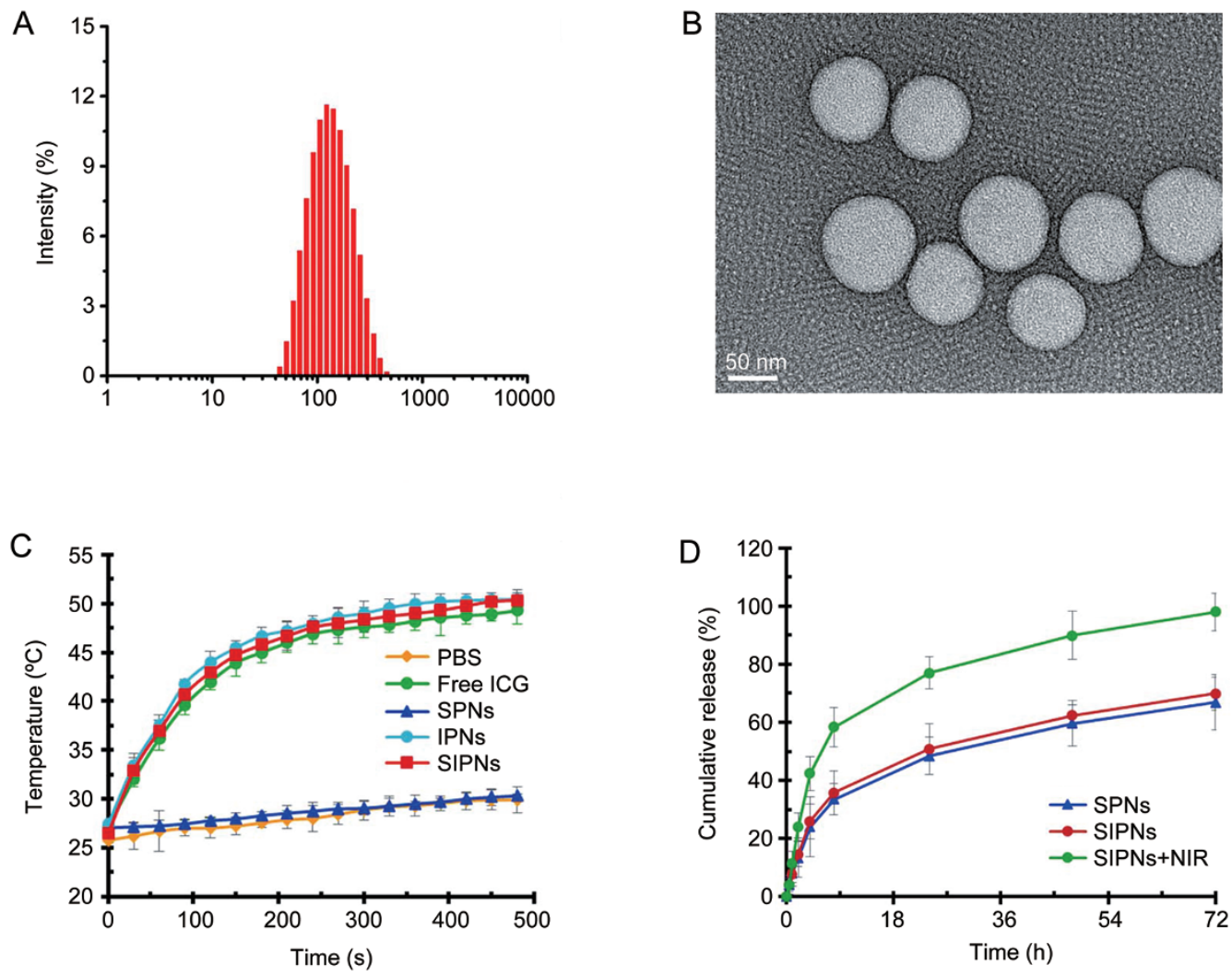

Figure 1. (A) The particle size distribution of the SIPNs is shown. (B) TEM images of SIPNs are represented. Scale bar=50 $\mathrm{nm}$. (C) The temperature elevation profiles of PBS, free ICG, SPNs, IPNs and SIPNs for $8 \mathrm{~min}$ are shown. (D) The in vitro release profiles of silibinin from the SPNs, SIPNs and SIPNs with NIR laser irradiation in PBS at $37^{\circ} \mathrm{C}$ are shown.

diation of $1.5 \mathrm{~W} \cdot \mathrm{cm}^{-2}$ for $8 \mathrm{~min}$ (Figure 1C). After irradiation exposure, the temperature of the free ICG, IPNs and SIPNs maximally increased to $49.2,50.8$ and $50.5^{\circ} \mathrm{C}$, respectively. In contrast, the SPNs only increased to $30.3^{\circ} \mathrm{C}$, similar to the PBS group. Therefore, the IPNs and the SIPNs could both positively contribute to the ablation of the cancerous cells.

The drug release experiments were used to evaluate the release behavior of the NPs (Figure 1D). The results showed that the SPNs and SIPNs exhibited a similar release profile during the test, indicating that the ICG loading in the nanoparticles did not change the release of the silibinin from the PCL/ lipid nanoparticles. The silibinin release was approximately $33.6 \%$ by $8 \mathrm{~h}$, indicating a lower release behavior. Upon irradiation with the NIR laser, the SIPNs displayed a burst release, especially during the first $8 \mathrm{~h}$. The silibinin release was $58.3 \%$, and the whole release was enhanced to $97.8 \%$. This indicated that the SIPNs could successfully achieve a higher temperature, inducing faster release of the loading drug under laser irradiation in vitro.

\section{Cellular uptake of SIPNs}

The cellular internalization of SIPNs was investigated using 4T1 breast cancer cells. The silibinin was replaced with Nile red, a hydrophobic fluorescent dye that has been widely used as a model drug to replace hydrophobic drugs ${ }^{[25,26]}$. Hoechst
33342 was used to stain the nuclei of the cells ${ }^{[27]}$. It was obvious that the free Nile red group exhibited a dim red fluorescence, indicating a lower uptake rate of the free drug in the $4 \mathrm{~T} 1$ cells in vitro (Figure 2). After the Nile red was loaded into the nanoparticles including SPNs and SIPNs, the stronger red fluorescence was distributed in the 4T1 cells in either SPNs or SIPNs, which suggested that SIPNs could be an effective drug delivery system that could accelerate the administration of the drugs into 4T1 cells. Moreover, the analysis did not show a significant difference between the red fluorescence in SPNs and SIPNs. However, after the cells treated with SIPNs were irradiated with an NIR laser, the red fluorescence was dramatically enhanced across a wide distribution of the cells. Therefore, the SIPNs could be utilized as a promising drug delivery system for enhancing the cellular internalization of the drugs, and the encapsulated ICG could induce hyperthermia along with an accelerated rate of drug release in the cells. These results together demonstrate that SIPNs could enhance the uptake of the nanoparticles and achieve hyperthermia, thereby inducing the rapid release of drugs at the target points under NIR laser irradiation in vitro, consistent with the detected drug release levels.

\section{Phototoxicity assay in vitro}

The in vitro cytotoxicity of free silibinin, PNs, SPNs, IPNs with 

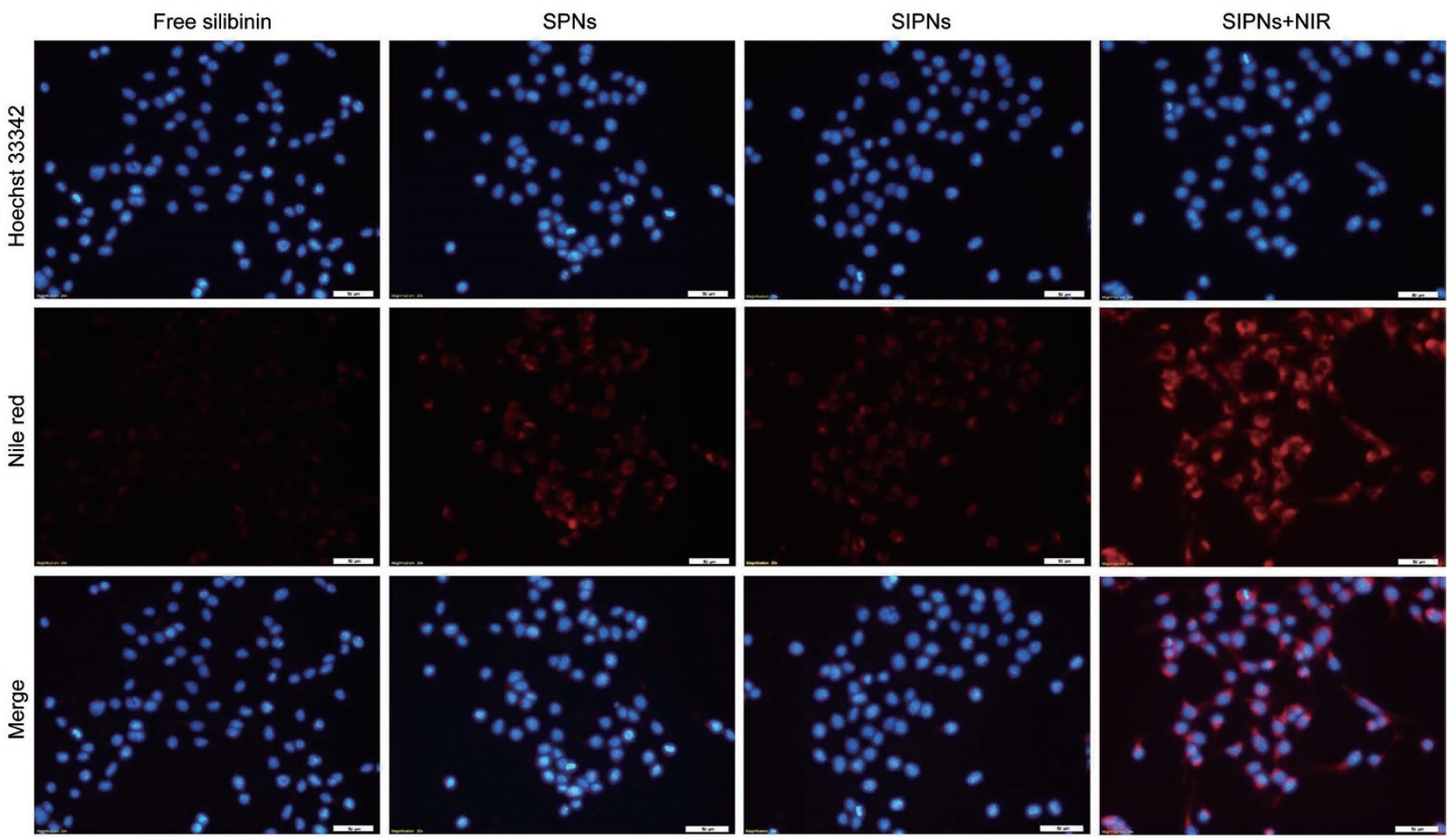

Figure 2. The cellular internalization of the nanoparticles by the $4 \mathrm{~T} 1$ cells after $2 \mathrm{~h}$ of incubation. The nucleus was stained with Hoechst 33342 (blue). The silibinin was replaced with Nile red. Scale bar $=50 \mu \mathrm{m}$.

NIR irradiation, SIPNs and SIPNs with NIR irradiation were evaluated by SRB assay. The silibinin-free PNs displayed negligible anti-proliferative effects on the 4T1 cells during the detected concentrations, indicating good biocompatibility of the nanocarriers (Figure 3A). The free silibinin group showed a relatively low cytotoxicity with over $80 \%$ cell viability at concentrations ranging from 1.0 to $100.0 \mu \mathrm{g} / \mathrm{mL}$ for $24 \mathrm{~h}$. The SPNs and SIPNs also exhibited a similarly low cytotoxicity among the tested concentrations, which may have occurred because the detected concentrations of silibinin could not cause obvious cytotoxicity of the $4 \mathrm{~T} 1$ cells. These results also suggested that the cell wound healing, migration and invasion effects of the free silibinin, SPNs and SIPNs had nothing to do with the anti-proliferative effects caused by these formulations on the cells. After irradiation with an NIR laser, the IPNs showed significantly increased cytotoxicity with an $\mathrm{IC}_{50}$ value of $20.8 \mu \mathrm{g} / \mathrm{mL}$, caused by the induced PTT effects of the ICG. For the SIPNs+NIR group, the anti-proliferative effects were slightly better than the IPNs with NIR irradiation, which could be attributed to the acceleration of the release of the loading drugs upon irradiation followed by the increased drug concentrations in the cells.

The phototoxicity of the nanoparticles that was induced by the NIR irradiation was further measured using the LIVE/ DEADViability/Cytotoxicity Kit according to the manufacturer's protocol (Figure 3B). The control group did not have any dead cells (red). There was a small difference between the SIPNs without NIR irradiation and the control group. Upon irradiation with the NIR laser, the IPNs and SIPNs both exhibited strong and wide red fluorescence, indicating that the tumor cells were damaged to death. Moreover, there was a negligible difference between dead cells induced by the IPNs and SIPNs, indicating that the silibinin was not responsible for destroying the cells, consistent with the cytotoxicity results shown above. Taken together, these results indicated that SIPNs could successfully achieve the PTT therapy under the NIR laser exposure.

\section{The wound healing assay}

The wound healing assay was used to evaluate the effects of the silibinin, PNs, SPNs, IPNs with NIR irradiation, SIPNs and SIPNs with NIR irradiation on inhibiting the motility of the $4 \mathrm{~T} 1$ cells (Figure 4 ). In the control group, the scratched spaces were barely evident, indicating that the 4T1 cells exhibited a high level of motility. The silibinin-free PNs groups and the control groups showed no obvious differences in the width of the scratches, suggesting that the nanocarriers did not inhibit the wound healing of the cells. In contrast, there was still an obvious wound in the cells treated with free silibinin. After the silibinin was loaded into the SPNs or SIPNs, the inhibitory effects were greater than in the case of free silibinin, and the wound healing rates were approximately 57.1\% (Figure 3B), which could be mainly due to the increased cellular uptake of the nanoparticles compared to the free drugs. Moreover, 

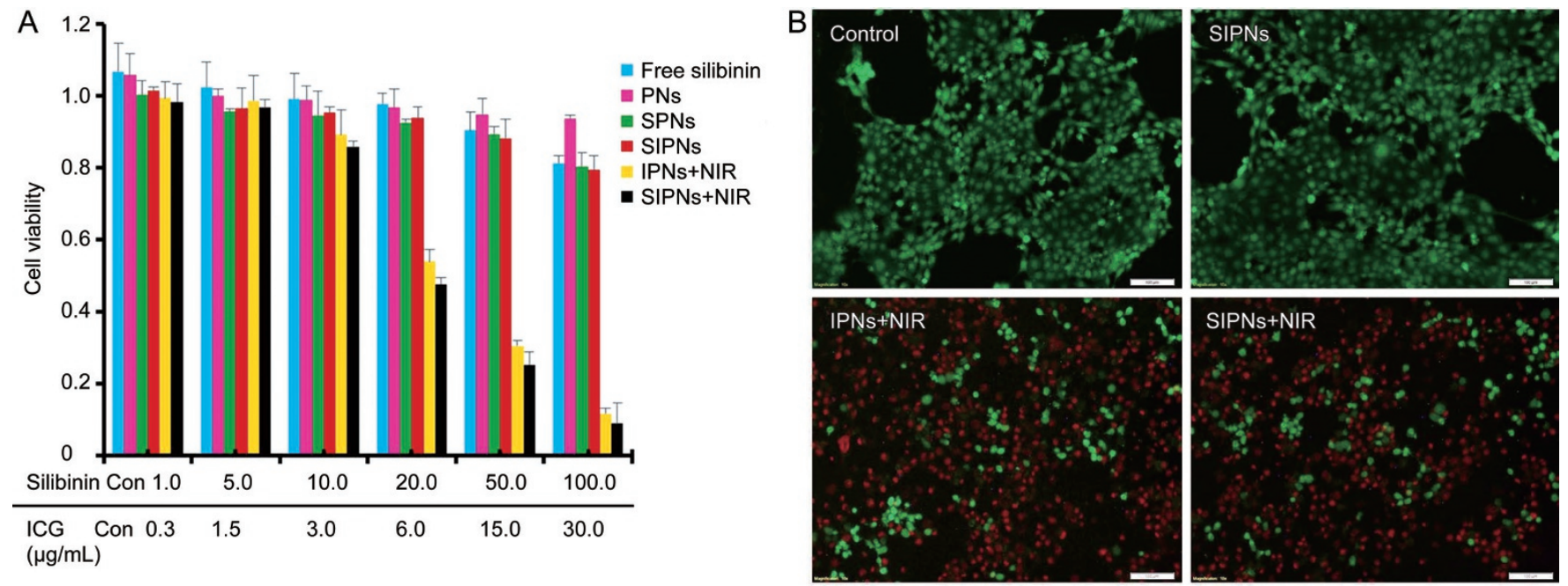

Figure 3. (A) The in vitro cytotoxicity effects of free silibinin, PNs, SPNs, SIPNs, IPNs with NIR irradiation and SIPNs with NIR irradiation at different concentrations on 4T1 cells after $24 \mathrm{~h}$ incubation are shown. (B) The photothermal cytotoxicity images of the nanoparticles in 4T1 cells are represented. The live cells were stained with calcein AM (green), and the dead cells were stained with EthD-1 (red). Scale bar=100 $\mu$ m.

A
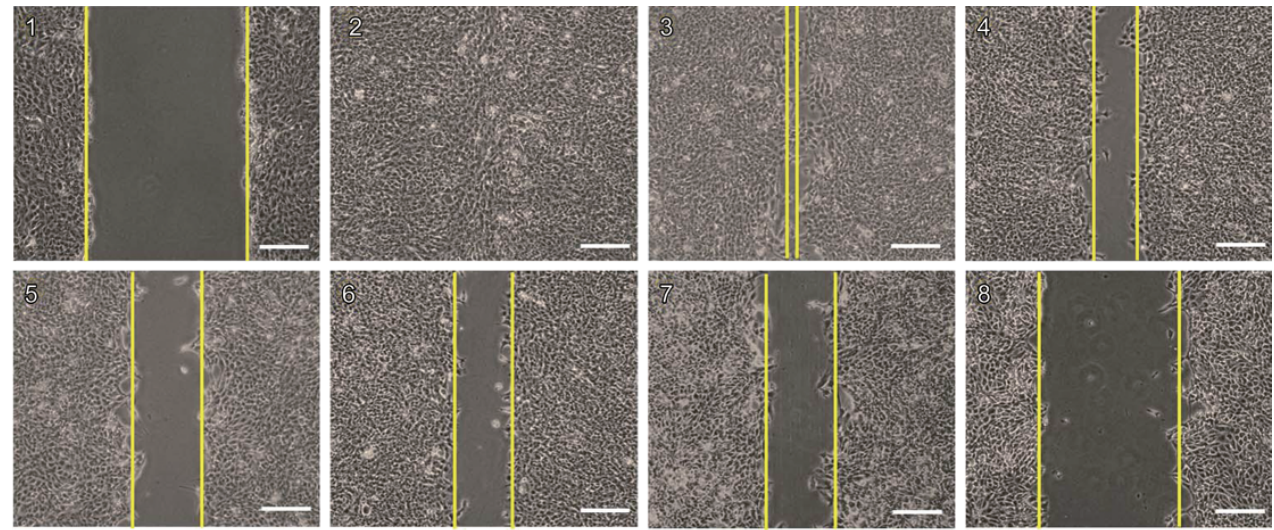

B

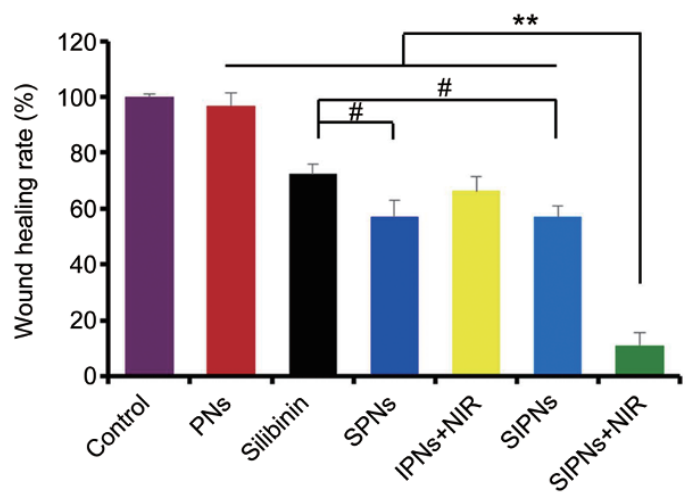

Figure 4. The inhibitory effects of the nanoparticles on the wound healing of 4T1 cells in vitro. (A) The images depict wound healing of cells (1) immediately following the scratches, (2) that received no treatments (control group) and that were treated with (3) PNs, (4) free silibinin, (5) SPNs, (6) IPNs with NIR irradiation, (7) SIPNs and (8) SIPNs with NIR irradiation. Scale bar=100 $\mu \mathrm{m}$. (B) The quantitative analysis of the wound healing rates of different nanoparticles-treated group was determined. The data are presented as the mean \pm SD $(n=3) .{ }^{* *} P<0.01$ vs SIPNs+NIR. ${ }^{*} P<0.05$ vs free silibinin. Two-tailed test. 
A
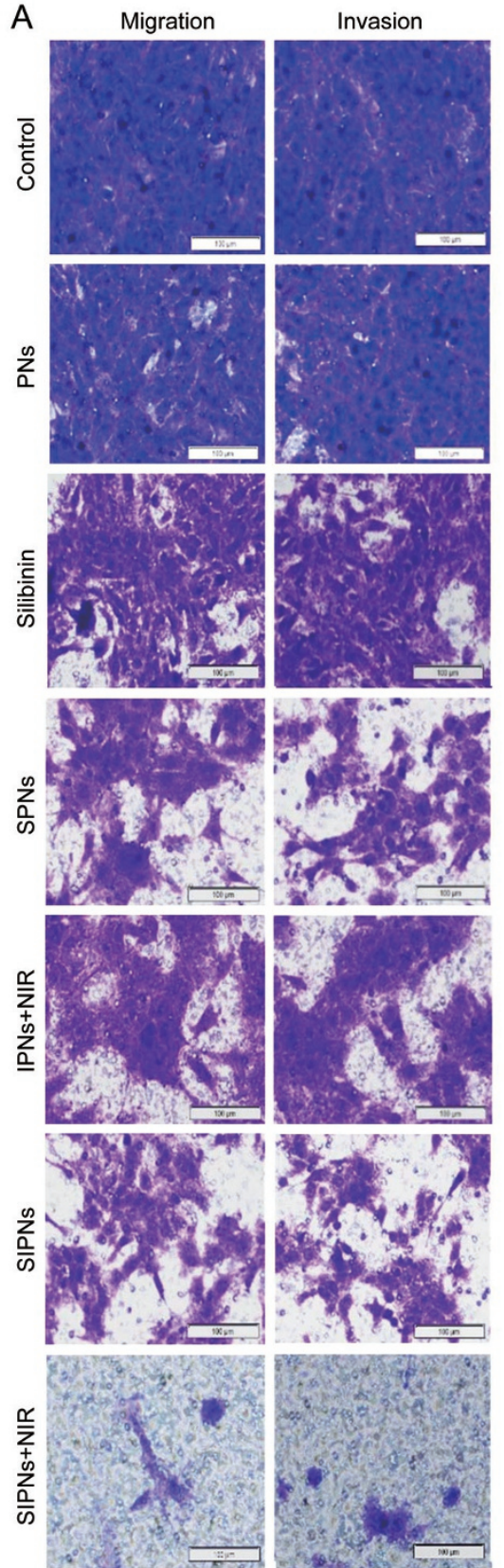

B

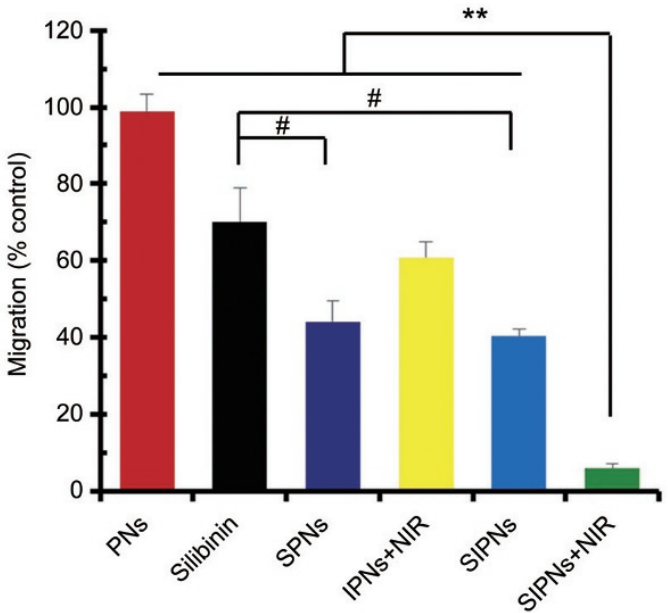

C

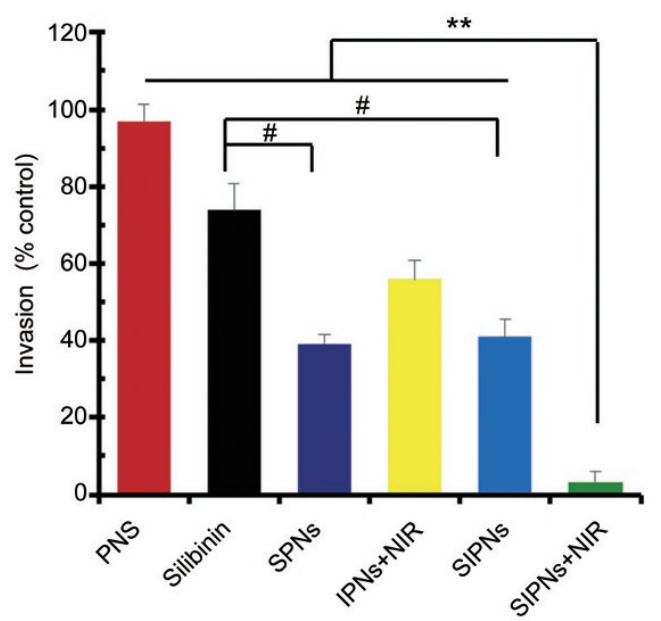

Figure 5. The inhibitory effects of the nanoparticles on the cell migration and invasion of 4T1 cells in vitro. (A) The representative images of the different formulations are shown. The cells were treated with PNs, free silibinin, SPNs, IPNs with NIR irradiation, SIPNs and SIPNs with NIR irradiation, respectively. Scale bar $=100 \mu \mathrm{m}$. The quantitative analysis of (B) migratory and (C) invasive cells from each group. The number of the control group was set as $100 \%$. The data are presented as the mean $\pm \operatorname{SD}(n=3)$. ${ }^{* *} P<0.01$ vs SIPNs+NIR. ${ }^{\#} P<0.05$ vs free silibinin. Two-tailed test.

the cells treated with IPNs plus NIR laser irradiation also displayed inhibitory effects and a wound healing rate of $66.2 \%$, indicating that the PTT could also suppress wound healing before inducing cell death. In the final formulation of SIPNs with NIR irradiation, the largest scratch wounds were evident, and the wound healing rate was $11.0 \%$. As a result, the wound healing results verified the high efficiency of SIPNs on inhibiting cell motility activities in response to NIR laser irradiation.

\section{In vitro migration and invasion}

It is well known that one of the critical steps for tumor metastasis is cell invasion, which includes the degradation of the extracellular matrix (ECM) barriers and the migration to distant organs ${ }^{[28]}$. Thus, the cell migration and invasion assays were applied to evaluate whether the nanoparticles could affect the migration and invasion of $4 \mathrm{~T} 1$ cells. The migration and invasion results were similar to each other (Figure 4). Compared to the control groups, the cells treated with 
PNs showed no significant difference in the cells on the lower chambers, indicating that the PNs could not inhibit the invasion of $4 \mathrm{~T} 1$ cells. The cells treated with free silibinin showed suppressed invasion with an invasion rate of approximately $73.8 \%$. While the silibinin in the nanoparticles containing SPNs and SIPNs showed significantly more inhibition with less invasion than the free silibinin group. The quantitative results also showed that the invasion rates of the SPNs and SIPNs were $39.3 \%$ and $41.0 \%$, respectively. The IPNs exposed to NIR laser irradiation also showed inhibitory effects compared to the control group, consistent with the wound healing results shown above. Finally, the SIPNs receiving the NIR laser exposure displayed dramatic anti-invasion effects with the appearance of a slight purple color in the obtained image and an invasion rate of only 3.2\%. This result further demonstrated the strong inhibitory effects caused by the combination of the faster photothermal-induced release of the drug and the suppression by the PTT that was generated from the loading ICG. Taken together, the cell migration and invasion results indicated that the SIPNs could be applied as an efficient drug delivery system for anti-metastasis in vitro.

\section{Discussion}

In the current study, SIPNs were fabricated by loading silibinin and ICG into PCL/lecithin nanoparticles to explore a new drug delivery system that aims to successfully inhibit tumor growth and metastasis in vitro. This unique drug delivery system could overcome the poor water solubility of silibinin, achieve a more rapid photothermal-induced release of the drugs in tumor cells and induce PTT effects to cooperatively inhibit the growth and metastasis of cancerous cells in vitro.

The SIPNs were successfully prepared, as demonstrated by the DLS results and the TEM images. It is well-known that tumor cells could be irreversibly destroyed under conditions of hyperthermia $\left(42-47^{\circ} \mathrm{C}\right)^{[29]}$. In the photothermal conversion assay, the high temperature of the SIPNs would be beneficial for the ablation of the tumor cells. Moreover, the induced hyperthermia could also increase the fluidity of the lipids ${ }^{[19]}$, which might contribute to an increased rate of release of the loading drug in the PCL/lecithin nanoparticles. The IPNs and SIPNs displayed a slightly higher temperature during the test, which could be due to the higher concentration of the condensed ICG in the nanoparticles than the free ICG and the entrapment of the induced thermal radiation in the nanoparticles with the stronger heat energy in the NPs. This phenomenon was also observed in previous reports ${ }^{[30]}$. After irradiation with an NIR laser, the SIPNs exhibited a fast release of silibinin, which could be mainly attributed to the photothermal-induced acceleration of the fluidity of the lecithin in addition to the increased drug release from the cores into the surrounding medium. The higher cellular uptake of the SIPNs further demonstrated the advantages of this delivery system. The NIR irradiation induces hyperthermia from the ICG, which increases the permeability of the nanoparticles for the loading drugs, accelerating the release of the drugs from the nanoparticles and also enhancing the permeability and fluidity of the cancer cell membranes of the $4 \mathrm{~T} 1$ cells $^{[31]}$ and increasing the cellular internalization of nanoparticles. Therefore, the SIPNs exposed to NIR laser irradiation exhibited the highest level of cell internalization. The anti-proliferative and phototoxicity results further demonstrated the excellent PTT effects of ICG loading in SIPNs, suggesting that SIPNs could inhibit the tumor cells growth in vitro. The cell wound healing and migration assays were then utilized to investigate the movement or migration ability of the tumor cells. The invasion assays were used to evaluate whether the treated tumor cells could degrade the matrigel and migrate into the lower chambers. The results detected in these assays indicated that SIPNs significantly suppressed the tumor cells metastasis in vitro, mainly due to the high concentration of the drugs caused by the faster hyperthermia-induced release of drugs under the NIR laser exposure. In addition, the PTT effects played a combinational role in suppressing the cells migration and invasion. It is notable that the nanocarriers used in this work were prepared by PCL, SPC and pluronic copolymer F68. The pluronic F68 is a typical amphiphilic polymer composed of hydrophilic poly(ethylene oxide) (PEO) blocks and hydrophobic poly(propylene oxide) blocks, in which the PEO segments are capable of preventing aggregation, recognizing the reticuloendothelial system (RES) and prolonging the circulation time of loading drugs in vivo ${ }^{[32]}$. Thus, SIPNs could also possess this long-circulating property in vivo.

In summary, the SIPNs inhibited tumor cell viability by the effects of PTT and by combinational treatments that address tumor cell growth and metastasis in vitro. Moreover, the rapid photothermal-induced release of the drugs in the tumor cells further enhanced the anti-metastasis effects. This new drug delivery system might be a promising platform for the future therapy of breast cancer metastasis in vivo.

\section{Acknowledgements}

The National Basic Research Program of China (No 2015CB932103, 2012CB932502), and the National Natural Science Foundation of China (№ 81521005, 81270047) are gratefully acknowledged for their financial support.

\section{Author contribution}

Hui-ping SUN performed the experiments and wrote the manuscript; Jing-han SU helped to perform the in vitro characterization of the obtained nanoparticles. Qing-shuo MENG, Qi YIN, Zhi-wen ZHANG, Hai-jun YU, Peng-cheng ZHANG, and Si-ling WANG analyzed the data. Ya-ping LI designed and supervised the study. All authors have read and approved the final manuscript.

\section{References}

1 Eckhardt BL, Francis PA, Parker BS, Anderson RL. Strategies for the discovery and development of therapies for metastatic breast cancer. Nat Rev Drug Discov 2012; 11: 479-97.

2 Chaffer CL, Weinberg RA. A perspective on cancer cell metastasis. Science 2011; 331: 1559-64.

3 Miller K, Wang M, Gralow J, Dickler M, Cobleigh M, Perez EA, et al. 
Paclitaxel plus bevacizumab versus paclitaxel alone for metastatic breast cancer. N Engl J Med 2007; 357: 2666-76.

4 Wulfkuhle JD, Liotta LA, Petricoin EF. Proteomic applications for the early detection of cancer. Nat Rev Cancer 2003; 3: 267-75.

5 Steeg PS. Tumor metastasis: mechanistic insights and clinical challenges. Nat Med 2006; 12: 895-904.

6 Xu P, Yin Q, Shen J, Chen L, Yu H, Zhang Z, et al. Synergistic inhibition of breast cancer metastasis by silibinin-loaded lipid nanoparticles containing TPGS. Int J Pharm 2013; 454: 21-30.

7 Xu P, Yu H, Zhang Z, Meng Q, Sun H, Chen X, et al. Hydrogen-bonded and reduction-responsive micelles loading atorvastatin for therapy of breast cancer metastasis. Biomaterials 2014; 35: 7574-87.

8 Veiseh O, Kievit FM, Ellenbogen RG, Zhang M. Cancer cell invasion: treatment and monitoring opportunities in nanomedicine. Adv Drug Del Rev 2011; 63: 582-96.

9 Jin ZH, Jin MJ, Jiang CG, Yin XZ, Jin SX, Quan XQ, et al. Evaluation of doxorubicin-loaded $\mathrm{pH}$-sensitive polymeric micelle release from tumor blood vessels and anticancer efficacy using a dorsal skin-fold window chamber model. Acta Pharmacol Sin 2014; 35: 839-45.

10 Punfa W, Yodkeeree S, Pitchakarn P, Ampasavate C, Limtrakul P. Enhancement of cellular uptake and cytotoxicity of curcumin-loaded PLGA nanoparticles by conjugation with anti-P-glycoprotein in drug resistance cancer cells. Acta Pharmacol Sin 2012; 33: 823-31.

11 Zhang L, Zhang S, Ruan SB, Zhang QY, He Q, Gao HL. Lapatinib-incorporated lipoprotein-like nanoparticles: preparation and a proposed breast cancer-targeting mechanism. Acta Pharmacol Sin 2014; 35 : 846-52.

12 Wang C, Sun X, Cheng L, Yin S, Yang G, Li Y, et al. Multifunctional theranostic red blood cells for magnetic-field-enhanced in vivo combination therapy of cancer. Adv Mater 2014; 26: 4794-802.

13 Guo L, Yan DD, Yang D, Li Y, Wang X, Zalewski O, et al. Combinatorial photothermal and immuno cancer therapy using chitosan-coated hollow copper sulfide nanoparticles. ACS Nano 2014; 8: 5670-81.

$14 \mathrm{He} \mathrm{X,} \mathrm{Bao} \mathrm{X,} \mathrm{Cao} \mathrm{H,} \mathrm{Zhang} \mathrm{Z,} \mathrm{Yin} \mathrm{Q,} \mathrm{Gu} \mathrm{W,} \mathrm{et} \mathrm{al.} \mathrm{Tumor-penetrating}$ nanotherapeutics loading a near-infrared probe inhibit growth and metastasis of breast cancer. Adv Funct Mater 2015; 25: 2831-9.

15 Hirsch LR, Stafford RJ, Bankson JA, Sershen SR, Rivera B, Price RE, et al. Nanoshell-mediated near-infrared thermal therapy of tumors under magnetic resonance guidance. Proc Natl Acad Sci U S A 2003; 100: 13549-54.

16 Wang D, Xu Z, Yu H, Chen X, Feng B, Cui Z, et al. Treatment of metastatic breast cancer by combination of chemotherapy and photothermal ablation using doxorubicin-loaded DNA wrapped gold nanorods. Biomaterials 2014; 35: 8374-84.

17 Guo C, Yin S, Yu H, Liu S, Dong Q, Goto T, et al. Photothermal ablation cancer therapy using homogeneous CsxW03 nanorods with broad near-infra-red absorption. Nanoscale 2013; 5: 6469-78.

18 Viger ML, Sheng W, Dore K, Alhasan AH, Carling CJ, Lux J, et al. Nearinfrared-induced heating of confined water in polymeric particles for efficient payload release. ACS Nano 2014; 8: 4815-26.

19 Zhao P, Zheng M, Luo Z, Gong P, Gao G, Sheng Z, et al. NIR-driven smart theranostic nanomedicine for on-demand drug release and synergistic antitumour therapy. Sci Rep 2015; 5: 14258.

20 Zheng $\mathrm{C}$, Zheng $\mathrm{M}$, Gong $\mathrm{P}$, Jia D, Zhang $\mathrm{P}$, Shi B, et al. Indocyanine green-loaded biodegradable tumor targeting nanoprobes for in vitro and in vivo imaging. Biomaterials 2012; 33: 5603-9.

21 Yavuz MS, Cheng Y, Chen J, Cobley CM, Zhang Q, Rycenga M, et al. Gold nanocages covered by smart polymers for controlled release with near-infrared light. Nat Mater 2009; 8: 935-9.

22 Huang XH, El-Sayed IH, Qian W, El-Sayed MA. Cancer cell imaging and photothermal therapy in the near-infrared region by using gold nanorods. J Am Chem Soc 2006; 128: 2115-20.

23 Moon HK, Lee SH, Choi HC. In vivo near-infrared mediated tumor destruction by photothermal effect of carbon nanotubes. ACS Nano 2009; 3: 3707-13.

24 Gao ZG, Tian L, Hu J, Park IS, Bae YH. Prevention of metastasis in a 4T1 murine breast cancer model by doxorubicin carried by folate conjugated pH sensitive polymeric micelles. J Control Release 2011; 152: 84-9.

25 Lee CS, Park W, Park SJ, Na K. Endolysosomal environment-responsive photodynamic nanocarrier to enhance cytosolic drug delivery via photosensitizer-mediated membrane disruption. Biomaterials 2013; 34: 9227-36.

26 Li K, Liu B. Polymer-encapsulated organic nanoparticles for fluorescence and photoacoustic imaging. Chem Soc Rev 2014; 43: 6570-97.

27 Duan X, Xiao J, Yin Q, Zhang Z, Yu H, Mao S, et al. Smart pH-sensitive and temporal-controlled polymeric micelles for effective combination therapy of doxorubicin and disulfiram. ACS Nano 2013; 7: 5858-69.

28 Wittekind C, Neid M. Cancer invasion and metastasis. Oncology 2005; 69 Suppl 1: 14-6.

29 Landry J, Samson S, Chretien P. Hyperthermia-induced cell-death, thermotolerance, and heat-shock proteins in normal, respirationdeficient, and glycolysis-deficient Chinese-hamster cells. Cancer Res 1986; 46: 324-27.

30 Zheng M, Yue C, Ma Y, Gong P, Zhao P, Zheng C, et al. Single-step assembly of DOX/ICG loaded lipid--polymer nanoparticles for highly effective chemo-photothermal combination therapy. ACS Nano 2013; 7: 2056-67.

31 Tang Y, Lei T, Manchanda R, Nagesetti A, Fernandez-Fernandez A, Srinivasan $S$, et al. Simultaneous delivery of chemotherapeutic and thermal-optical agents to cancer cells by a polymeric (PLGA) nanocarrier: an in vitro study. Pharm Res 2010; 27: 2242-53.

32 Zhang W, Shi Y, Chen Y, Hao J, Sha X, Fang X. The potential of pluronic polymeric micells encapsulated with paclitaxel for the treatment of melanoma using subcutaneous and pulmonary metastatic mice models. Biomaterials 2011; 32: 5934-44. 